\title{
Assessing drug safety in human tissues - what are the barriers?
}

\author{
Anthony Holmes, Frank Bonner and David Jones
}

The drug development process is long and expensive. High attrition rates due to safety liabilities observed in the clinic, but that were not identified during nonclinical development, are a major contributor to the escalating costs and reduced productivity of the industry (Organs-on-chips at the frontiers of drug discovery. Nat. Rev. Drug Discov. 14, 248-260 (2015)) $)^{1}$. This has called into question the utility of current preclinical safety-testing paradigms - which rely predominantly on animal models, including rodents, dogs and nonhuman primates - and led to demands for more-predictive tools.

The development and use of human tissue-based assays provides an obvious alternative to the current approaches used to predict human pharmacological responses. However, despite this, very little drug development seems to be conducted using human tissues. We know from discussions with the UK Medicines and Healthcare Products Regulatory Agency (MHRA) that some companies do use these models for supporting internal early development decision-making processes, but this is on a relatively small scale and the data are rarely included in submissions to the international regulatory agencies.

The National Centre for the Replacement, Refinement and Reduction of Animals in Research (NC3Rs) and the MHRA are working together to evaluate the application and increase the uptake of human tissue-based approaches, to replace the use of animals and provide more-predictive tools to determine the safety of drugs entering clinical studies. We have convened an expert working group that comprises 25 individuals from a number of organizations (including academic organizations, companies and international regulators) to generate an evidence base to support these efforts (see Supplementary information S1 (table) for a list of members of the group). Our initial priority has been to assess the current use of human tissue through a survey of the international safety pharmacology community; however, the longer-term goal is the application of human tissue-based approaches to safety assessment in the broadest sense. Here, we present and discuss the results of this survey.

\section{Survey characteristics}

The working group developed the survey to gather information on general attitudes towards the use of human tissue-based approaches for generating safety data and record details of specific assays currently being used for safety assessment (see Supplementary information S2 (table) for the survey). The survey was distributed to the global safety-assessment community through various organizations, including the European Federation of Pharmaceutical Industries and Associations (EFPIA), the British Toxicology Society (BTS), the British Pharmacological Society (BPS) and EuroTox. The survey was completed by 27 individuals or companies who were primarily from large pharmaceutical companies (52\%), with the remaining respondents divided equally between small pharmaceutical companies, biotechnology companies and academic institutions. The survey included information on 28 specific human-tissue assays currently being used for safety assessment.

For the purpose of the survey, human tissue was broadly defined as any material derived from human sources, including organs, tissues, cells, biofluids and so on. Assays in which cells of human origin are used as vehicles for the introduction of channels were not included in the questionnaire.

\section{Survey results}

General attitudes to the use of human tissues for safety pharmacology studies. Almost half (48\%) of the respondents currently use human tissue-based assays for safety pharmacology studies. However, the proportion of respondents who are aware of human tissue-based approaches currently under evaluation within their organization or who intend to use human tissue-based assays in the future increases to $63 \%$ and $93 \%$, respectively. This indicates a growing appetite for the development and use of human tissue models for safety assessment and shows that it is timely to consider opportunities for supporting researchers in this endeavour.

Just over half of respondents (51\%) considered the greater relevance of human tissue models to human safety as the main motivation for using these models. Other sources of motivation included the poor predictivity of animal models (18\%), the lack of suitable animal models (10\%) and the lower cost of running in vitro assays (6\%) (FIG. 1). There is clear demand for human tissue models across the core and non-core battery studies within safety pharmacology, as well as in toxicology. Perhaps unsurprisingly, given the impact on drug development, it is in cardiovascular safety assessment that there is greatest demand for human models (56\%), with fairly consistent demand across the remaining core and non-core battery endpoints, as well as the toxicology endpoints.

\section{Barriers to wider uptake of human tissue-} based approaches. Respondents were asked what they considered to be the main barriers to the uptake of human tissue approaches. More than one answer could be selected and the responses suggest that it is not one particular factor that acts as a barrier to wider adoption but a number of factors, which on their own may be resolvable but, when combined, may prove to be an overwhelming challenge. These barriers can be grouped into categories and are summarized in TABLE 1.

Information on specific human tissue assays currently adopted for safety pharmacology studies. Twelve companies (8 pharmaceutical and 4 biotechnology and/or contract research

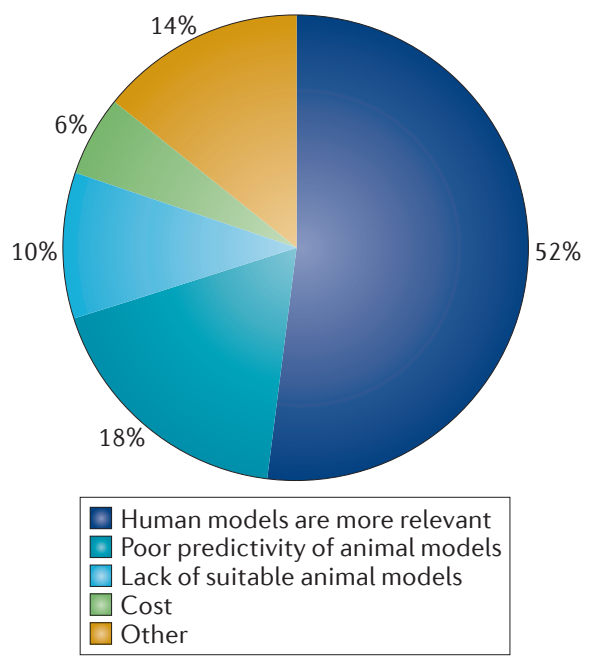

Figure 1 | The motivations behind adopting human tissue-based approaches for safety pharmacology studies. Respondents were asked what they considered to be their main motivation for the use of human tissue in safety pharmacology studies. Respondents were able to select more than one option and the percentage of the total number of responses is shown in the figure for each option. Seven respondents (14\% of the total) selected 'other' from the list. 


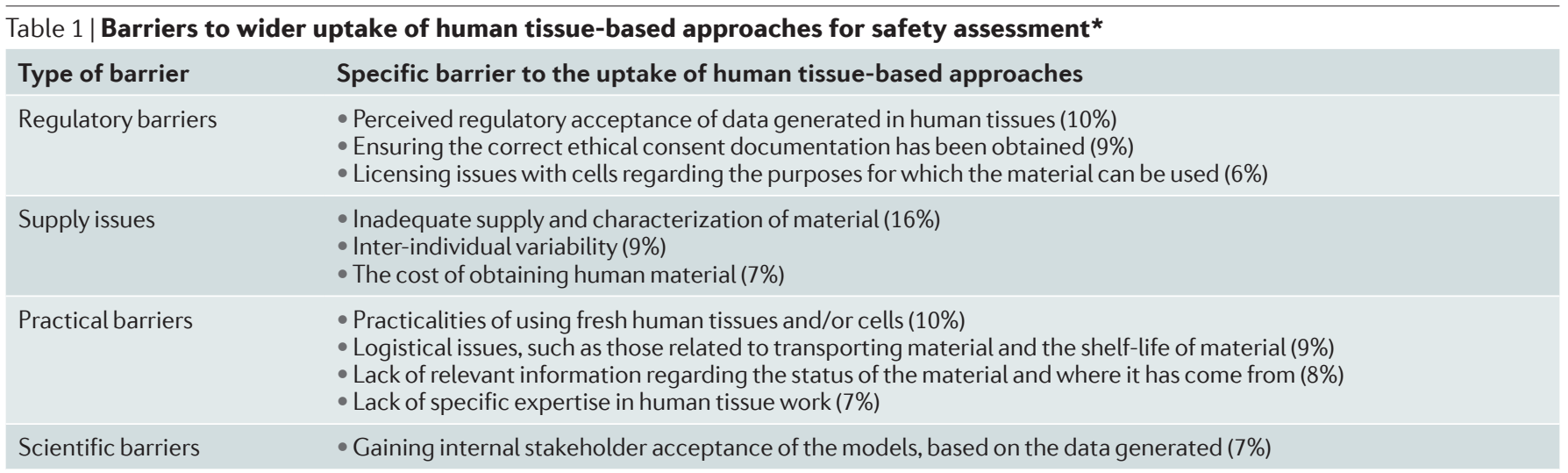

*Barriers can be grouped into categories depending on whether they are regulatory barriers, barriers because of supply issues, practical barriers or scientific barriers. Respondents were able to select more than one option and the percentage of the total number of responses is shown for each barrier in brackets. Three respondents ( $2 \%$ of the total) selected 'other' from the list of barriers.

organizations (CROs)) submitted data on 28 different human tissue assays that are currently being used as part of their safety pharmacology process. Most of these assays are ex vivo tissue preparations (29\%) and nearly half of them (46\%) are currently used to investigate cardiovascular liabilities. Fourteen per cent (14\%) of assays are used for respiratory assessment, but no human tissue assays are used for assessing central nervous system liabilities. The remaining responses were for non-core battery studies in liver (11\%), gastrointestinal (7\%) and other systems, including studies of the immune response (14\%) and skin (4\%). Other assay platforms adopted included mixed (14\%) and single cell type (14\%) $2 \mathrm{D}$ cell cultures, $3 \mathrm{D}$ cultures of multiple cells (11\%), cells in suspension (14\%) and tissue slices (11\%). There was no trend associated with which organ system these assay platforms were used to investigate. None of the respondents is currently using bioreactor or microfluidic approaches, despite recent advances in these technologies ${ }^{1-3}$.

Companies that responded to the survey are generally using these human tissue assays early in drug development (before in vivo studies (39\%) or concurrently with or after in vivo studies (47\%)) to improve mechanistic understanding (25\%) or compound selection (32\%), or to demonstrate safety in a human model (39\%). These assays are generally carried out because they are a part of that company's routine safety pharmacology assessment (24\%) or because of concerns from regulatory in vitro studies, in vivo studies or clinical trials (64\%). This is contradictory to the provided data regarding when these studies are conducted; however, this may be due to historical knowledge from previous studies or similar compound classes.
A positive result in these safety-assessment assays has a range of consequences, with some companies dropping the compound (20\%), others conducting further in vitro or in vivo studies (34\%), and $29 \%$ of respondents using the data to provide better insight into the mechanism of action of the compound being tested. However, it is worth noting that half of these $29 \%$ selected this as an additional consequence of the positive result to others already selected. Other consequences of a positive result related to clinical trials, with either further trials being required (3\%) or specific recommendations being required for monitoring in clinical trials (9\%).

\section{Discussion}

The survey has highlighted that the use of human tissues for safety assessment is not widespread, but there is increasing interest and demand for such approaches across safety pharmacology and toxicology disciplines. This is generally because human tissues are believed to provide a more relevant model system than current animal models $-64 \%$ of respondents agreed that clinical findings relating to adverse effects in humans are poorly predicted by the animal models currently used to generate organspecific safety data. Companies currently using human tissue models use them on a case-by-case basis early in drug development for de-risking compound selection and reducing the number of compounds entering regulatory in vivo studies later in development. However, these data are not generally included in clinical trial applications, the two most common reasons cited being: first, that the regulators do not request the data and, if included, these data may result in further questions and demands for additional studies, and second, that the respondents are not confident in the level of validation the model has been through. This is despite $93 \%$ of respondents confirming their model had been through some form of validation process.

Regulators are keen to support companies in developing and applying new non-animal approaches that can improve drug development, and they should not be seen as a barrier to this progress. However, to maximize the benefit of the guidance that regulators can provide, industry needs to engage with them early and regularly as data emerge and the technology grows to demonstrate its utility and justify its use. New guidance from the European Medicines Agency to support this process through a 'safe harbour' approach has been drafted (see Further information).

Many of the other barriers highlighted in TABLE 1 may look substantial on the surface, but they could potentially be overcome by sharing data, experiences and information regarding what resources and networks are available for accessing human tissue, the practical approaches to studies using human tissues and the logistical requirements to support this, and how to work within the potential confines of using commercially sourced human tissue for drug development. The development of human-based non-animal technologies is a rapidly evolving field, with advances being made in the development of tissue engineering, microfluidics and organ-on-chip technologies for many of the organ systems relevant to the safety assessment of new drugs. The safetyassessment community needs to do more to connect with these technology developers through initiatives such as Horizon 2020, the open innovation platform $\underline{\text { CRACK IT }}$ and the Innovate UK Non-Animal 
Technologies platform (see Further information) to help to shape the research and development of new technologies to meet their needs.

The current survey illustrates the views of a representative proportion of the safetyassessment community, and we would encourage others with relevant experience to complete the survey and build on this body of data. The survey data provide important evidence that a more concerted effort is needed to support scientists in adopting human tissue-based approaches to support drug development and the $3 \mathrm{Rs}$ (that is, the replacement, reduction and refinement of animals in research). The safety-assessment community needs to come together to share information on how these barriers can be overcome, and it is important that regulators and study directors play their part in supporting this. Additionally, there needs to be a meeting of minds between caregivers and researchers to ensure that as much as possible of the human tissue surplus to healthcare needs that is collected in hospitals is made available to the research community. Recognizing these needs, the NC3Rs is developing tools to help researchers to access the relevant information to expedite the wider adoption of human tissue-based models in basic research and drug development.

Anthony Holmes is at the National Centre for the Replacement, Refinement and Reduction of Animals in Research (NC3Rs), London NW1 2BE, UK.

Frank Bonner is at Stem Cells for Safer Medicines (SC4SM), London SW1E 6QT, UK.

David Jones is at the Medicines and Healthcare products Regulatory Agency (MHRA), London SW 1 W 9SZ, UK. Correspondence to A.H. e-mail: anthony.holmes@nc3rs.org.uk doi: $10.1038 /$ nrd4662

1. Esch, E. W. Bahinski, B. \& Huh, D. Organs-on-chips at the frontiers of drug discovery. Nat. Rev. Drug Discov. 14, 248-260 (2015).

2. Williamson, A., Singh, S., Fernekorn, U. \& Schober, A. The future of the patient-specific body-on-a-chip. Lab. Chip 13, 3471-3480 (2013).
3. Chan, C. Y. et al. Accelerating drug discovery via organs-on-chips. Lab. Chip 13, 4697-4710 (2013).

Acknowledgements

The authors would like to acknowledge the contribution made by the members of the NC3Rs and MHRA joint working group (see supplementary information for membership) for developing the survey and analysing the data, the organizations that helped to disseminate the survey, and the respondents who took the time to complete the survey.

Competing interests

The authors declare no competing interests.

\section{FURTHER INFORMATION}

CRACK IT: technologies for better science:

http://www.crackit.org.uk/

Guideline on regulatory acceptance of 3R (replacement,

reduction, refinement) testing approaches:

http://www.ema.europa.eu/docs/en GB/document library/

Scientific guideline/2014/10/WC500174977.pdf

Horizon 2020: the EU framework programme for research

and innovation: http://ec.europa.eu/programmes/

horizon2020/en

Innovate UK Non-Animal Technologies platform:

https:// connect.innovateuk.org/web/non-animal-

technologies/overview

SUPPLEMENTARY INFORMATION

See online article: $\underline{\mathrm{S} 1}$ (table) $\mid \underline{\mathrm{S}}$ (table)

ALL LINKS ARE ACTIVE IN THE ONLINE PDF 